\title{
Levels and values of circulating endothelial progenitor cells, soluble angiogenic factors, and mononuclear cell apoptosis in liver cirrhosis patients
}

\author{
Chih-Hung Chen ${ }^{1}$, Li-Teh Chang ${ }^{2}$, Wei-Chih Tung ${ }^{3}$, Yung-Lung Chen ${ }^{4}$, Chia-Lo Chang ${ }^{5}$, Steve Leu ${ }^{6}$, \\ Cheuk-Kwan Sun ${ }^{7}$, Tzu-Hsien Tsai ${ }^{4}$, I-Ting Tsai ${ }^{7}$, Hsueh-Wen Chang ${ }^{8}$ and Hon-Kan Yip ${ }^{4,6^{*}}$
}

\begin{abstract}
Background: The roles of circulating endothelial progenitor cell (EPC) and mononuclear cell apoptosis (MCA) in liver cirrhosis (LC) patients are unknown. Moreover, vascular endothelial growth factor (VEGF) and stromal cell-derived factor (SDF)-1a are powerful endogenous substances enhancing EPC migration into circulation. We assessed the level and function of EPCs [CD31/CD34 $\left.\left(E_{1}\right), K D R / C D 34\left(E_{2}\right), C X C R 4 / C D 34\left(E_{3}\right)\right]$, levels of MCA, VEGF and SDF-1a in circulation of LC patients.
\end{abstract}

Methods: Blood sample was prospectively collected once for assessing EPC level and function, MCA, and plasma levels of VEGF and SDF-1a using flow cytometry and enzyme-linked immunosorbent assay (ELISA), respectively, in $78 \mathrm{LC}$ patients and 25 age- and gender-matched healthy controls.

Results: Number of EPCS $\left(E_{1}, E_{2}, E_{3}\right)$ was lower (all $p<0.0001$ ), whereas SDF-1a level and MCA were higher $(p<0.001)$ in study patients compared with healthy controls. Number of EPCS $\left(E_{2}, E_{3}\right)$ was higher but MCA was lower (all $p<0.05$ ) in Child's class A compared with Child's class B and C patients, although no difference in VEGF and SDF-1a levels were noted among these patients. Chronic hepatitis $B$ and esophageal varices bleeding were independently, whereas chronic hepatitis C, elevated aspartate aminotransferase (AST), and decompensated LC were inversely and independently correlated with circulating EPC level (all $p<0.03$ ). Additionally, angiogenesis and transwell migratory ability of EPCs were reduced in LC patients than in controls (all $p<0.001$ ).

Conclusion: The results of this study demonstrated that level, angiogenic capacity, and function of circulating EPCS were significantly reduced, whereas plasma levels of SDF-1a and circulating MCA were substantially enhanced in cirrhotic patients.

Keywords: Liver cirrhosis, Circulating endothelial progenitor cells, Angiogenesis factors, Cellular apoptosis

\section{Background}

Since multitudinous studies have shown that circulating endothelial progenitor cells (EPCs) are essential for neoangiogenesis and the endothelial repair process, they

\footnotetext{
*Correspondence: han.gung@msa.hinet.net

${ }^{4}$ Division of Cardiology, Department of Internal Medicine, Kaohsiung Chang Gung Memorial Hospital and Chang Gung University College of Medicine, 123, Ta Pei Road, Niao Sung Hsiang, Kaohsiung Hsien 83301, Taiwan

${ }^{6}$ Center for Translational Research in Biomedical Sciences, Kaohsiung Chang Gung Memorial Hospital and Chang Gung University College of Medicine, Kaohsiung, Taiwan

Full list of author information is available at the end of the article
}

have been employed for the treatment of both experimental and clinical organ ischemia in various settings over the past few years [1-6]. Growing data have demonstrated that EPC migration into circulation and homing to ischemic tissues/organs are strongly associated with an increase in circulating levels of chemokines [7], cytokines [8], recombinant human erythropoietin (EPO) [9], and some specific medications such as statins [10] or angiotensin converting enzyme inhibitors (ACEIs) [11], as well as extracorporeal shock wave therapy [12]. Additionally, angiogenesis in malignant tumors and 
metastasis have also been reported to be associated with an increased number of circulating EPCs $[13,14]$. Besides, vascular endothelial growth factor (VEGF) and stromal cell-derived factor- 1 alpha (SDF-1 $\alpha$ ), two powerful soluble angiogenesis factors, have been shown to play a crucial role in enhancing EPC migration from bone marrow into circulation [15]. Conversely, decreased circulating number of EPCs has been found to correlate to the prevalence of risk factors for coronary artery disease $[16,17]$, recurrent ischemic stroke [18], and advanced heart failure [19].

Liver cirrhosis (LC) of divergent etiologies is at high risk of developing into hepatocellular carcinoma (HCC) $[20,21]$. Moreover, abundant studies have shown that not only serum levels of soluble angiogenic factors are notably increased in patients with LC and those with HCC, but they are also useful biomarkers for predicting the prognostic outcome in these patient populations [22-24]. Surprisingly, the role of circulating EPCs in the clinical setting of LC has seldom been investigated [25]. Besides, the link between circulating number of EPC and plasma level of VEGF and SDF- $1 \alpha$ is currently unclear in LC patients. Furthermore, although progression of chronic liver disease to $\mathrm{LC}$ is known to involve distinctive apoptosis and death of hepatocytes, there is a lack of in-depth scrutiny regarding the role of circulating mononuclear cell apoptosis (MCA) in modulating the severity of chronic liver disease. Of particular importance is that the function of EPC in LC patients has not been reported. In this study, the above issues were addressed by applying flow cytometric and ELISA analysis to determine the number and function of EPCs, the distinctive role of circulating MCA, and the relationship between EPC biomarkers and plasma levels of VEGF and SDF- $1 \alpha$ in LC patients.

\section{Methods}

\section{Patient enrollment and blood sampling}

The estimated sample size of 86 patients was based on the effective size with an $\alpha$ value of 0.05 , a power of $80 \%$, a $0.7 \%$ difference in circulating level of EPCs between study patients and healthy control subjects, and standard deviations of $0.7 \%$ in normal control subjects and $1.0 \%$ in study patients. A $12.0 \%$ rate of protocol violations was assumed. The calculation of sample size for specific objective was based on our recent report [18]. The expected $0.7 \%$ was based on our previous study of EPC surface marker of CD31/CD34 on 138 patients [18].

To circumvent other potential influences on measurement of circulating level of EPCs, patients with one or more of the followings were excluded: recent surgery or trauma during the preceding 2 months, malignancy, history of febrile disorders, acute or chronic inflammatory disease other than LC during the study period, history of autoimmune diseases with or without immunosuppressive therapy, and prior myocardial infarction with an onset of $<3$ months.

Between September 2009 and December 2010, 88 patients who presented to the Department of Gastroenterology at Kaohsiung Chang Memorial Hospital with the diagnosis of LC were recruited. Patients were enrolled either from out patient department $(n=7$, all of them were Child's class A patients) (followed-up for LC) or during hospitalization $(\mathrm{n}=71)$ for severe jaundice, ascites with hypoalbuminemia for plasma transfusion, acute aspartate aminotransferase (AST) and alanine aminotransferase (ALT) elevation, anti-hepatitis B (entecavir) treatment, anti-hepatitis $\mathrm{C}$ (interferon) treatment, esophageal varices ligation or esophageal varices bleeding $(\mathrm{n}=10)$. After excluding 10 patients due to infection or refusing to participate in the study, blood sampling was performed in totally 78 consecutive patients. All blood sampling was collected prior to blood transfusion.

Twenty-five age- and gender-matched healthy controls were also studied. Informed consent was obtained from all study subjects. The study protocol was approved by the Institutional Review Committee on Human Research at Kaohsiung Chang Gang Memorial Hospital.

\section{Subgroup analysis of the patients}

According to the degree of decompensation in liver function, the study patients were categorized into Child's class $\mathrm{A}, \mathrm{B}$, and $\mathrm{C}$ to investigate whether the circulating levels of EPCs, MCA, and plasma levels of SDF- $1 \alpha$ and VEGF were different among these patients.

\section{Ultrasonographic examination and definitions}

Ultrasonographic scans were performed by experienced hepatologists at our institute using an ultrasound system with a $3.5 \mathrm{MHz}$ convex probe (SSA-340, SSA-370, Aplioxu-700 Toshiba, Tokyo, Japan; SSD 2000, Aloka, Tokyo, Japan; HDI 5000, ATL Ultrasound, Bothell, USA; Preirus Hi-vision Hitachi, Tokyo, Japan).

Chronic hepatitis $\mathrm{C}$ was defined as detectable serum antibody to hepatitis $\mathrm{C}$ virus (anti-HCV), and chronic hepatitis B was defined as detectable serum hepatitis B viral surface antigen (HBsAg). The definitions and diagnoses of HCC, LC, and the degree of decompensation were based on previous reports [26-30].

\section{Blood sampling and assessment of circulating EPC level by flow cytometry}

Blood samples were obtained once in ward or in the outpatient department for the study group and also once for the healthy control subjects. Twenty milliliters of blood was drawn from the antecubital vein $(10 \mathrm{~mL}$ for EPC analysis and another $10 \mathrm{~mL}$ for MCA assessment). 
Mononuclear cells (MNCs) were then isolated by density-gradient centrifugation of Ficoll 400 (Ficoll-Pla$\mathrm{que}^{\mathrm{TM}}$ plus, Amersham Biosciences, Sweden) as previously described [31]. The isolated MNCs were washed twice with phosphate buffer solution (PBS) and centrifuged before incubation with $1 \mathrm{~mL}$ blocking buffer (BSA) for 30 minutes at $4{ }^{\circ} \mathrm{C}$. Cell viability of $>95.0 \%$ was noted in each group.

It has been reported that EPCs are characterized by surface expression of CD34, CD31, CXCR4 and kinase insert domain-conjugating receptor (KDR) antigens $[9,12,18,19,31,32]$. In this study, EPCs were defined as cells with co-expression of CD34, endothelial cell (EC) lineage-markers [KDR, vascular endothelial (VE)-cadherin, CD31, and CXCR4 according to previous reports $[9,12,18,19,31,32]$ and our recent study $[18]$.

EPCs in peripheral blood were identified by flow cytometry using the technique of double staining as depicted in our recent report [18]. To determine the EPC surface markers of CD31/CD34 $\left(E_{1}\right), \operatorname{KDR} / \mathrm{CD} 34 \quad\left(\mathrm{E}_{2}\right)$ and CXCR4/CD34 $\left(E_{3}\right)$, MNCs $\left(4 \times 10^{5}\right)$ were incubated for 30 minutes at $4{ }^{\circ} \mathrm{C}$ in a dark room with monoclonal antibodies against KDR (Sigma), fluorescein isothiocyanate (FITC)-conjugated CD34, phycoerythrin (PE)-conjugated CD31, and CXCR4 (Becton Dickinson). Control ligand (IgG-PE conjugate) was used to detect possible nonspecific association and define a threshold for glycoprotein binding. For analysis of KDR, MNCs were further incubated with goat anti-mouse antibody conjugated with PE. After staining, the MNCs were fixed in $1 \%$ paraformaldehyde. Quantitative two-color flow cytometric analysis was performed using a fluorescence-activated cell sorter (FACSCalibur ${ }^{\mathrm{TM}}$ system; Beckman). Each analysis included 30,000 cells (i.e., mononuclear cells) per sample. The assays for EPCs $\left(\mathrm{E}_{1-3}\right)$ in each sample were performed in duplicate and the mean levels were reported. The results were expressed as percentage (\%) of total mononuclear cells per sample.

Intra-assay variability based on repeated measurement of the same blood sample was low with a mean coefficient of variance of $4.6 \%$ in LC patients and $4.1 \%$ in healthy control subjects.

\section{Mononuclear cell culture, differentiation of endothelial cell phenotype, angiogenesis, and measurement of total tubular length}

To evaluate the degree of angiogenesis, another $10 \mathrm{~mL}$ of blood was drawn randomly from $6 \mathrm{LC}$ patients $(2$ in Child's Class A, B and C, respectively) and 6 normal subjects with age match, respectively. The protocol and procedure of cell culture and the determination of angiogenesis were based on our recent report [33]. Briefly, the MNCs were isolated by density-gradient centrifugation of Ficoll 400, followed by cultivation in differential endothelial cell culture medium (endothelial cell basal medium-2, Cambrex) with $10 \%$ fetal bovine serum (FBS), $50 \mathrm{U} / \mathrm{mL}$ penicillin, $50 \mu \mathrm{g} / \mathrm{mL}$ streptomycin and $2 \mathrm{mmol} / \mathrm{L}$ L-glutamine (Invitrogen) with VEGF and basic fibroblast growth factor $(10 \mathrm{ng} / \mathrm{ml})$ plated on gelatin-coated tissue culture flasks and incubated at $37{ }^{\circ} \mathrm{C}$ with $5 \% \mathrm{CO}_{2}$ for 21 days. Culture medium was changed every 48 hours. By day 21, cells with spindleshaped and cobblestone-like phenotype typical of endothelial cells were found attached on the plate.

The cells with endothelial cell phenotype were then plated in 96-well plates at $1.0 \times 10^{4}$ cells/well in $150 \mu \mathrm{L}$ serum-free M199 culture medium mixed with $50 \mu \mathrm{L}$ cold Matrigel (Chemicon international) for 24 hours using passage $2 \mathrm{EPCs}$ incubated at $37{ }^{\circ} \mathrm{C}$ in $5 \% \mathrm{CO}_{2}$. Three random microscopic images $(200 \mathrm{x})$ were taken from each well for counting cluster, tube, and network formations with the mean values obtained. Both cumulative and mean tube lengths were calculated by ImagePro Plus software (Media Cybernetics).

\section{Transwell migratory assay}

To investigate the transwell migratory ability of EPCs ( $n=6$ in each experiment), transwell membranes $(5 \mu \mathrm{m}$; Costar, Germany) were coated on both sides with fibronectin $(2.5 \mu \mathrm{g} / \mathrm{mL}$; Roche, Mannheim, Germany) overnight at $4{ }^{\circ} \mathrm{C}$. The experimental protocol was also based on that of our recent report [32]. EPCs $\left(1 \times 10^{5}\right.$ cells/ well) were resuspended in M199 medium (Gibco, Carlsbad, CA, USA) containing 0.5 FBS (Gibco, Carlsbad, CA, USA) and incubated in the upper chamber at $37{ }^{\circ} \mathrm{C}$ in $5 \% \mathrm{CO}_{2}$ and allowed to migrate for 18 hours toward the lower chamber which was filled with M199 containing $20 \%$ FBS. Cells remaining on the upper surface of the transwell membranes were mechanically removed and cells that had migrated to the lower surface were fixed with $4 \%$ formaldehyde. For cell quantification, nuclei of the migrated cells were stained with DAPI. Cells migrating into the lower chamber were counted in 5 random microscopic fields using a fluorescence microscope (Olympus, Tokyo, Japan) with the software ImagePro Plus (Media Cybernetics, Bethesda, MD, USA).

\section{Determination of annexin V level by flow cytometry}

Flow cytometric analysis using double staining of annexin $\mathrm{V}$ and propidium iodide (PI) is a simple and popular method for the identification of apoptotic cells [i.e. early phase (annexin $\mathrm{V}+/ \mathrm{PI}-$ ) and late phase (annexin $\mathrm{V}+/ \mathrm{PI}+$ ) of apoptosis].

Ten milliliters of peripheral blood MNCs were isolated by density gradient centrifugation of Ficoll 400 (FicollPlaque $^{\mathrm{TM}}$ plus, Amersham Biosciences, Sweden). Cell viability of $>95.0 \%$ was noted in each group. The protocol and procedure were based on our recent report [33]. 
The annexin V kit (Pharmingen, Becton Dickinson, San José, CA, USA) was used for apoptosis analysis according to the manufacturer's instructions. The supernatant was decanted and the pellet was resuspended in $200 \mu \mathrm{L}$ of $1 \mathrm{x}$ annexin V binding buffer. Cells were incubated with $2 \mu \mathrm{L}$ of annexin V-FITC and $5 \mu \mathrm{L}$ of propidium iodide (PI) for 15 minutes at room temperature in the dark. Finally, $500 \mu \mathrm{L}$ of $1 \mathrm{x}$ annexin V Binding Buffer was added, followed by immediate flow cytometric analysis. Identical to the analysis of EPCs, each analysis included 30,000 cells (i.e., mononuclear cells) per sample. The assays for MCA in each sample were performed in duplicate and the mean levels were reported. The results were expressed as percentage (\%) of total mononuclear cells per sample.

Intra-assay variability based on repeated measurements of the same blood sample was low with a mean coefficient of variance less than $4.2 \%$ in both study patients and normal subjects.

\section{Measurement of plasma levels of VEGF and SDF-1a using ELISA}

Plasma concentrations of VEGF and SDF-1 $\alpha$ were assessed using VEGF and SDF- $1 \alpha$ antibody ELISA kit $(R \& D)$, respectively according to the manufacturer's protocol.

\section{Statistical analysis}

Data were expressed as means $\pm \mathrm{SD}$. Continuous data between two groups were analyzed using Wilcoxon rank sum test, and categorical data were analyzed by chisquare or Fischer exact test. For determining statistical significance among the three groups, continuous data were analyzed using Kruskal-Wallis test, followed by Wilcoxon rank sum test with Bonferroni's correction. Statistical analysis was performed using SAS statistical software for Windows version 8.2 (SAS institute, Cary, $\mathrm{NC}$ ). A p value of $<0.05$ was considered statistically significant.

\section{Results}

Table 1 shows the baseline characteristics and laboratory findings of study patients and healthy control subjects. The age, gender, white blood cell (WBC) count, and serum creatinine level did not differ between the two groups. However, the circulating level of EPCs [CD31/CD34( $\left.E_{1}\right)$, $\left.\operatorname{KDR} / C D 34\left(E_{2}\right), \quad C X C R 4 / C D 34\left(E_{3}\right)\right]$, hemoglobin level, platelet count, and serum albumin concentration were remarkably lower in study patients than those in control subjects. By contrast, the serum levels of AST, ALT, and plasma level of SDF- $1 \alpha$ were substantially increased in study patients compared with those in control subjects, whereas plasma VEGF level was similar between the two groups. Additionally, flow cytometry revealed a notable enhancement of early and late MCA in LC patients than in healthy control subjects in the present study (Table 1).

The incidences of male gender, diabetes mellitus, hypertension, and current smoking did not differ among Child's class A, B and C patients (Table 2). The incidences of chronic hepatitis B, chronic hepatitis C, history of esophageal varices (EV) bleeding, ascites on sonographic examination, and portal venous hypertension were similar among the three groups of patients. However, patients in the Child's class $C$ were younger than the other two groups. Additionally, the incidences of decompensated LC and portal venous hypertension were remarkably higher in patients in Child's class $C$ than those in Child's class A and B, and notably higher in patients in Child's class B than those in Child's class A. Furthermore, the incidences of current smoking and splenomegaly were significantly higher in Child's class B and $C$ patients than those in Child's class A, but they showed no difference between those in Child's class B and $\mathrm{C}$.

Flow cytometric analysis (Table 3) demonstrated that the circulating number of $E_{2}$ and $E_{3}$ was significantly increased in Child's class A patients than those in Child's class $B$ and $C$, but the number of $E_{1}, E_{2}$ and $E_{3}$ did not differ between those in Child's class $B$ and $C$.

To elucidate the impact of decompensation of liver function on MCA in circulation, flow cytometric analysis using the technique of double staining for annexin $\mathrm{V}$ and PI was performed for each patient. The results showed that early MCA was similar among the three groups of the patients, whereas late MCA was substantially lower in those in Child's class A than those in Child's class B and C. No significant difference, however, was noted between patients in Child's class B and C.

Laboratory findings demonstrated no significant differences in hemoglobin, WBC count, platelet count, serum levels of creatinine, AST, ALT, and $\alpha$-fetoprotein among the three groups of patients. On the other hand, prothrombin time and serum level of total bilirubin were significantly lower in Child's class A than in Child's class $\mathrm{B}$ and $\mathrm{C}$ patients. These two parameters, however, did not differ between Childs' class B and C patients. Paradoxically, serum albumin level was notably lower in Child's class A than in Child's class B and C patients.

The plasma level of SDF-1 $\alpha$ was similar among the three groups of patients. In addition, although no significant difference in plasma level of VEGF was noted between Child's class $B$ and $C$ patients, it was significantly lower in Child's class A than in Child's class B and $\mathrm{C}$ patients.

To compare the number of circulating EPCs and plasma levels of VEGF and SDF- $1 \alpha$ among LC patients with $(n=66)$ or without HCC $(n=12)$ and the healthy control $(\mathrm{n}=25)$ subjects, subgroup analysis was 
Table 1 The baseline Characteristics of Study Patients and Normal Controls

\begin{tabular}{|c|c|c|c|}
\hline Variables & Study patients $(n=78)$ & Normal control $(n=25)$ & p value* \\
\hline Age (yrs) & $54.8 \pm 13.7$ & $54.1 \pm 10.5$ & 0.927 \\
\hline Male gender & $71.8 \%(56)$ & $68.0 \%(17)$ & 0.716 \\
\hline \multicolumn{4}{|l|}{ EPC surface markerst } \\
\hline CD31/CD34 (\%)\$ & $1.01 \pm 0.57$ & $1.37 \pm 0.49$ & 0.005 \\
\hline KDR/CD34 (\%)* & $1.08 \pm 0.63$ & $1.37 \pm 0.65$ & 0.035 \\
\hline CXCR4/CD34 (\%): & $1.13 \pm 0.66$ & $1.54 \pm 0.64$ & 0.001 \\
\hline WBC count $\left(\times 10^{3} / \mathrm{dl}\right)$ & $8.5 \pm 11.9$ & $6.0 \pm 1.5$ & 0.575 \\
\hline Hemoglobin (g/dl) & $10.9 \pm 2.4$ & $14.9 \pm 1.3$ & $<0.0001$ \\
\hline Platelet count $\left(\times 10^{4} / \mathrm{dl}\right)$ & $10.8 \pm 6.3$ & $22.4 \pm 5.5$ & $<0.0001$ \\
\hline Creatinine (mg/dl) & $1.00 \pm 0.60$ & $0.91 \pm 0.20$ & 0.806 \\
\hline Albumin (g/dl) & $3.0 \pm 0.6$ & $4.5 \pm 0.3$ & $<0.0001$ \\
\hline AST (U/L) & $97.8 \pm 107.1$ & $24.6 \pm 6.4$ & $<0.0001$ \\
\hline $\mathrm{ALT}(\mathrm{U} / \mathrm{L})$ & $68.1 \pm 123.0$ & $29.3 \pm 17.4$ & 0.005 \\
\hline VEGF (pg/ml) & $55.4 \pm 58.0$ & $49.9 \pm 27.8$ & 0.286 \\
\hline SDF-1a (pg/ml) & $2256.3 \pm 705.8$ & $1502.4 \pm 370.7$ & $<0.0001$ \\
\hline Early apoptosis (\%) & $18.01 \pm 11.66$ & $6.16 \pm 2.83$ & $<0.0001$ \\
\hline Late apoptosis (\%) & $3.28 \pm 3.86$ & $1.19 \pm 0.99$ & 0.0008 \\
\hline
\end{tabular}

Data expressed as mean \pm SD or \% (No)

$\mathrm{ALT}=$ alanine aminotransferase; $\mathrm{AST}=$ aspartate aminotransferase; $\mathrm{EPC}=$ endothelial progenitor cell; SDF = stromal cell-derived factor; $\mathrm{VEGF}=$ vascular endothelial cell growth factor; $\mathrm{WBC}=$ white blood cell.

* by Wilcoxon rank sum test.

† indicates EPC surface markers with double stains.

$\$$ The expected $0.7 \%$ was based on our previous study [18] on EPC surface markers (CD31/CD34). The expected difference (i.e., $0.7 \%$ ) has not been reached for CD31/CD34 (or KDR/CD34, CXCR4/CD34) in Table 1.

performed (Figure 1A). The number of circulating $E_{2}$ did not differ among LC patients and healthy controls. Additionally, the numbers of circulating $\mathrm{E}_{1}$ were similar between LC patients with or without HCC. Furthermore, the number of circulating $E_{1}$ did not differ between LC with HCC and healthy controls, but it was significantly lower in LC patients without HCC than in healthy controls. Moreover, the circulating number of $\mathrm{E}_{3}$ did not

Table 2 Clinical Characteristics and Abdominal Ultrasonographic Findings of 78 Study Patients

\begin{tabular}{|c|c|c|c|c|}
\hline Variables & Child's class A $(n=9)$ & Child's class B $(n=26)$ & Child's class C $(n=43)$ & p value* \\
\hline Age (yrs) & $59.9 \pm 11.3^{a}$ & $59.6 \pm 14.4^{a}$ & $50.8 \pm 12.7^{b}$ & 0.030 \\
\hline Male gender & $55.6 \%(5)$ & $65 . \%(17)$ & $79.1 \%(34)$ & 0.234 \\
\hline Diabetes mellitus & $33.3 \%(3)$ & $46.2 \%(12)$ & $20.9 \%(9)$ & 0.089 \\
\hline Hypertension & $22.2 \%(2)$ & $11.5 \%(3)$ & $2.3 \%(1)$ & 0.0503 \\
\hline Current smoking & $11.1 \%(1)^{a}$ & $73.1 \%(19)^{b}$ & $48.8 \%(21)^{b}$ & 0.006 \\
\hline Chronic hepatitis B & $55.6 \%(5)$ & $38.5 \%(10)$ & $27.9 \%(12)$ & 0.251 \\
\hline Chronic hepatitis C & $11.1 \%(1)$ & $30.8 \%(8)$ & $10(23.3 \%)$ & 0.513 \\
\hline Previous EV bleeding & $22.2 \%(2)$ & $30.8 \%(8)$ & $46.5 \%(20)$ & 0.295 \\
\hline Hepatocellular carcinoma & $0 \%(0)$ & $23.1 \%(6)$ & $14.0 \%(6)$ & 0.286 \\
\hline Liver cirrhosis & $100.0 \%(9)$ & $100.0 \%(26)$ & $100.0 \%(43)$ & 1.000 \\
\hline Ascitis & $22.2 \%(2)$ & $50.0 \%(13)$ & $58.1 \%(25)$ & 0.136 \\
\hline Decompensated liver cirrhosis & $11.1 \%(1)^{a}$ & $38.5 \%(10)^{b}$ & $67.4 \%(29)^{c}$ & 0.002 \\
\hline Splenomegaly & $22.2 \%(2)^{a}$ & $76.9 \%(20)^{b}$ & $67.4 \%(29)^{b}$ & 0.015 \\
\hline Portal vein hypertension & $11.1 \%(1)^{\mathrm{a}}$ & $30.8 \%(8)^{b}$ & $53.5 \%(23)^{c}$ & 0.028 \\
\hline
\end{tabular}

Data expressed as mean \pm SD or \% (No).

EV, esophageal varices.

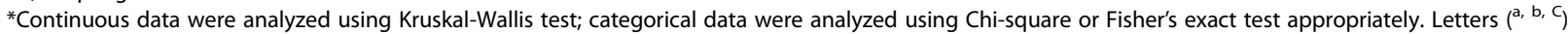
indicate significant difference (at 0.05 level) (by Wilcoxon rank sum test with Bonferroni's correction). 
Table 3 Flow Cytometric Analysis and Laboratory Findings of 78 Study Patients

\begin{tabular}{|c|c|c|c|c|}
\hline Variables & Child's class A $(n=9)$ & Child's class B $(n=26)$ & Child's class C $(n=43)$ & p value* \\
\hline \multicolumn{5}{|c|}{ EPC surface makers (double stains) } \\
\hline CD31/CD34 (\%) & $1.23 \pm 0.44$ & $0.90 \pm 0.52$ & $1.03 \pm 0.61$ & 0.279 \\
\hline KDR/CD34 (\%) & $1.60 \pm 0.51^{\mathrm{a}}$ & $0.97 \pm 0.63^{b}$ & $1.03 \pm 0.61^{b}$ & 0.013 \\
\hline CXCR4/CD34 (\%) & $1.49 \pm 0.56^{\mathrm{a}}$ & $1.20 \pm 0.63^{b}$ & $1.00 \pm 0.61^{b}$ & 0.043 \\
\hline Early apoptosis (\%) & $13.5 \pm 8.9$ & $16.8 \pm 10.8$ & $19.7 \pm 12.5$ & 0.281 \\
\hline Late apoptosis (\%) & $1.66 \pm 1.62^{\mathrm{a}}$ & $3.12 \pm 4.50^{b}$ & $3.67 \pm 3.7^{b}$ & 0.046 \\
\hline Hemoglobin (g/dl) & $12.2 \pm 2.2$ & $10.1 \pm 2.5$ & $11.1 \pm 2.2$ & 0.110 \\
\hline WBC count $\left(\times 10^{3} / \mathrm{dl}\right)$ & $5.0 \pm 2.1$ & $8.7 \pm 12.5$ & $8.8 \pm 12.3$ & 0.700 \\
\hline Platelet count $\left(\times 10^{4} / \mathrm{dl}\right)$ & $13.6 \pm 11.9$ & $11.0 \pm 5.5$ & $10.3 \pm 5.7$ & 0.930 \\
\hline Creatinine (mg/dl) & $0.84 \pm 0.23$ & $0.93 \pm 0.34$ & $1.08 \pm 0.74$ & 0.805 \\
\hline Albumin (g/dl) & $2.54 \pm 0.33^{\mathrm{a}}$ & $2.89 \pm 0.54^{b}$ & $2.87 \pm 0.58^{b}$ & 0.005 \\
\hline AST (U/L) & $50.0 \pm 23.6$ & $114.0 \pm 152.1$ & $98.0 \pm 80.4$ & 0.097 \\
\hline $\operatorname{ALT}(\mathrm{U} / \mathrm{ml})$ & $45.0 \pm 32.5$ & $83.6 \pm 195.2$ & $63.5 \pm 67.7$ & 0.361 \\
\hline Prothrombin time (sec) & $11.0 \pm 0.7^{\mathrm{a}}$ & $13.6 \pm 3.9^{b}$ & $13.2 \pm 2.2^{b}$ & 0.006 \\
\hline a-fetoprotein (ng/ml) & $8.3 \pm 3.2$ & $75.8 \pm 223.5$ & $1187.5 \pm 6495.3$ & 0.430 \\
\hline Total bilirubin (mg/dl) & $0.8 \pm 0.2^{\mathrm{a}}$ & $3.4 \pm 3.2^{b}$ & $4.8 \pm 6.4^{b}$ & 0.0002 \\
\hline VEGF (pg/ml) & $25.0 \pm 11.4^{\mathrm{a}}$ & $54.1 \pm 22.4^{b}$ & $62.6 \pm 59.8^{b}$ & 0.039 \\
\hline SDF-1a (pg/ml) & $2030.1 \pm 446.4$ & $2246.1 \pm 808.0$ & $2309.8 \pm 687.6$ & 0.350 \\
\hline
\end{tabular}

differ between LC patients with HCC and healthy control subjects. Interestingly, this biomarker $\left(\mathrm{E}_{3}\right)$ in $\mathrm{LC}$ patients without $\mathrm{HCC}$ was not only notably lower than in healthy subjects but it was also significantly lower than in LC patients with HCC (Figure 1A). The plasma level of VEGF showed no difference either between LC patients with and without HCC, or between LC patients without HCC and healthy subjects. This biomarker, however, was notably elevated in patients with HCC compared with that in healthy subjects (Figure 1B). Additionally, the plasma level SDF-1 $\alpha$ did not differ between LC patients with or without HCC, or between LC patients without $\mathrm{HCC}$ and healthy subjects, but it showed significantly higher in LC patients with HCC than in healthy subjects (Figure 1C). These findings imply that LC patients with HCC might have higher ability than those with LC alone to secrete SDF-1 $\alpha$ and VEGF that, in turn, enhanced the mobilization of EPCs from bone marrow to circulation.

To identify the independent predictors of increased circulating level of EPCs (Table 4), a number of enrollment variables were utilized (Tables 2 and 3). Multiple linear regression analysis demonstrated that chronic hepatitis B and EV bleeding were significantly and independently associated with increased circulating level of
EPCs. By contrast, hepatitis C, decompensated LC, and serum AST level were inversely and independently predictive of decreased circulating level of EPCs.

To compare the angiogenesis ability and EPC function between study patients and healthy control subjects, MNC culture for EPC was performed (Figure 2). Interestingly, the results showed that the cluster formation $(\mathrm{p}<0.001)$, tubular formation $(\mathrm{p}<0.0001)$, and network formation $(p<0.0001)$ were remarkably lower in LC patients than in healthy control subjects. In addition, the accumulative tubular length $(\mathrm{P}<0.0001)$ was notably reduced in LC patients than in healthy control subjects. Furthermore, the number of transwell migratory EPCs was substantially lower in LC patients as compared with those in healthy control subjects $(\mathrm{p}<0.0001)$.

\section{Discussion}

Several striking implications were noted in this study that investigated the number and function of circulating of EPCs, plasma levels of VEGF, SFD-1 $\alpha$, and MCA in circulation. First, the circulating levels of SDF- $1 \alpha$ as well as the degree of both early and late MCA were remarkably higher in LC patients compared with those in healthy control subjects. Second, the circulating number of EPCs was notably lower in LC patients than that in 

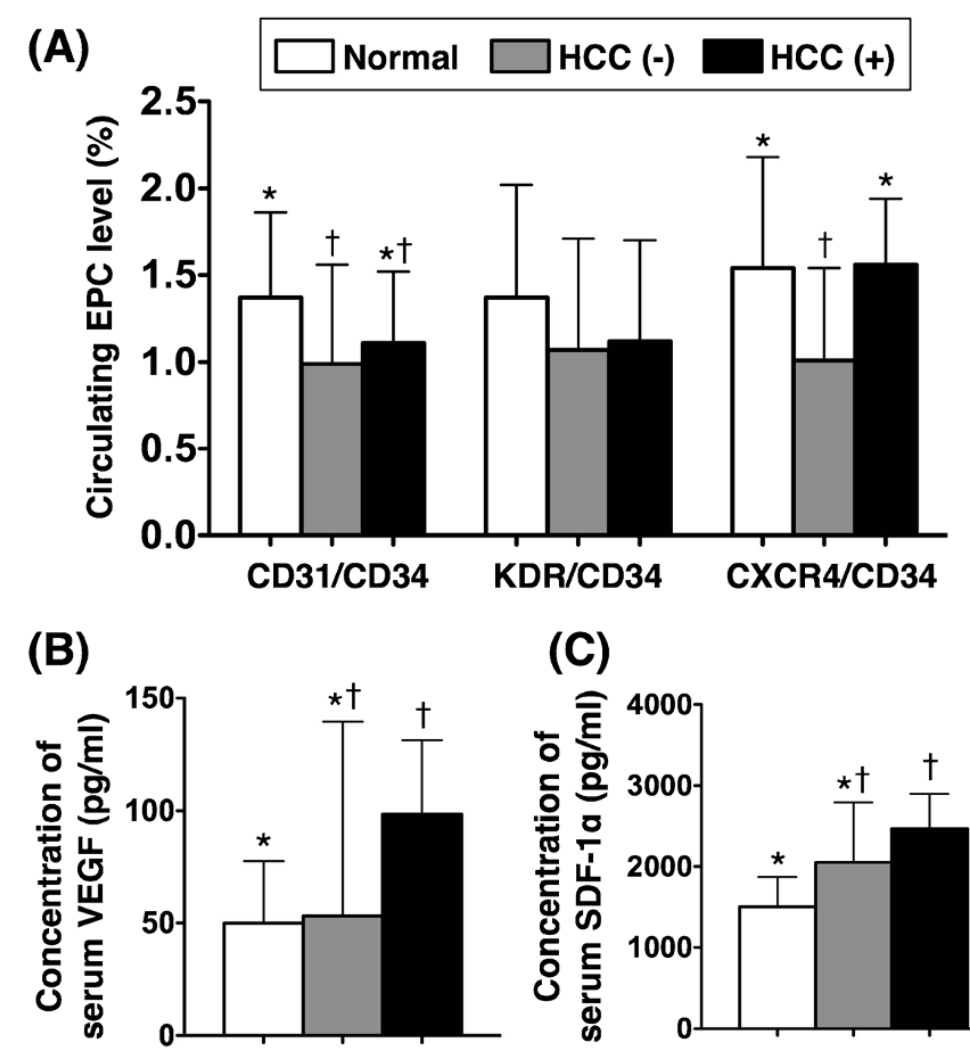

(C)

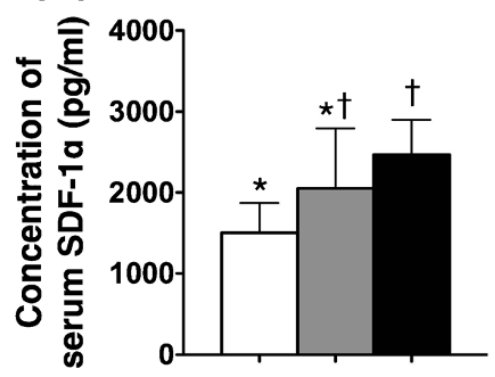

Figure 1 Flow Cytometric and ELISA Analysis among Patients With and Without HCC and in Normal Control Subjects. A) Circulating number of endothelial progenitor cells (EPCs). For CD31/CD34+ cells, ${ }^{*}$ vs.,$+ p<0.04$. For CXCR4+/CD34+ cells, * vs. t, $p<0.03$. HCC $(-)=$ without hepatocellular carcinoma $(n=66)$; HCC $(+)=$ with hepatocellular carcinoma $(n=12)$. B) Plasma level of vascular endothelial growth factor (VEGF). ${ }^{*}$ vs. $\dagger, p<0.035$. C) Plasma level of stromal cell-derived factor (SDF)-1a. ${ }^{*}$ vs. $\dagger, p<0.04$. Symbols $\left({ }^{*},+\right)$ indicate significant difference (at 0.05 level) (by Wilcoxon rank sum test with Bonferroni's correction).

normal control subjects. Third, the circulating number of EPCs was markedly decreased, whereas the degree of late MCA in circulation was significantly increased in Child's class B and C patients compared with those in Child's class A.

Surprisingly, while the association between increased serum levels of soluble angiogenic factors and chronic liver disease and HCC has been extensively investigated, circulating EPC level in LC patients has seldom been reported [25]. One important finding in the current study is that the number of circulating EPCs was notably reduced in patients with LC compared to that in ageand gender-matched healthy control subjects. Of particularly interest is that, when compared with Child's class A patients, this biomarker was further decreased in those in Child's class B and C. Our results, therefore,

Table 4 Multiple Stepwise Linear Regression Analysis of Independent Predictors of Increased Circulating Level of EPCs*

\begin{tabular}{|c|c|c|c|c|c|c|}
\hline \multirow[t]{2}{*}{ Variables } & \multicolumn{2}{|c|}{ CD31/CD34 } & \multicolumn{2}{|c|}{ KDR/CD34 } & \multicolumn{2}{|c|}{ CXCR4/CD34 } \\
\hline & Coefficient $\beta$ & $p$ value & Coefficient $\beta$ & $p$ value & Coefficient $\beta$ & $p$ value \\
\hline$\overline{\text { Age }}$ & -0.009 & 0.059 & & & & \\
\hline Hepatitis B & 0.340 & 0.011 & 0.380 & 0.008 & & \\
\hline Hepatitis C & & & -0.332 & 0.035 & & \\
\hline EV bleeding & & & 0.291 & 0.028 & & \\
\hline Liver cirrhosist & 0.352 & 0.087 & & & -0.708 & 0.003 \\
\hline AST & & & -0.001 & 0.006 & & \\
\hline
\end{tabular}

$\mathrm{AST}=$ aspartate aminotransferase; $\mathrm{EPC}=$ endothelial progenitor cells; $\mathrm{EV}=$ esophageal varicis.

* Variables of Tables 2 and 3 were enrolled for analysis.

† indicated decompensated liver cirrhosis. 

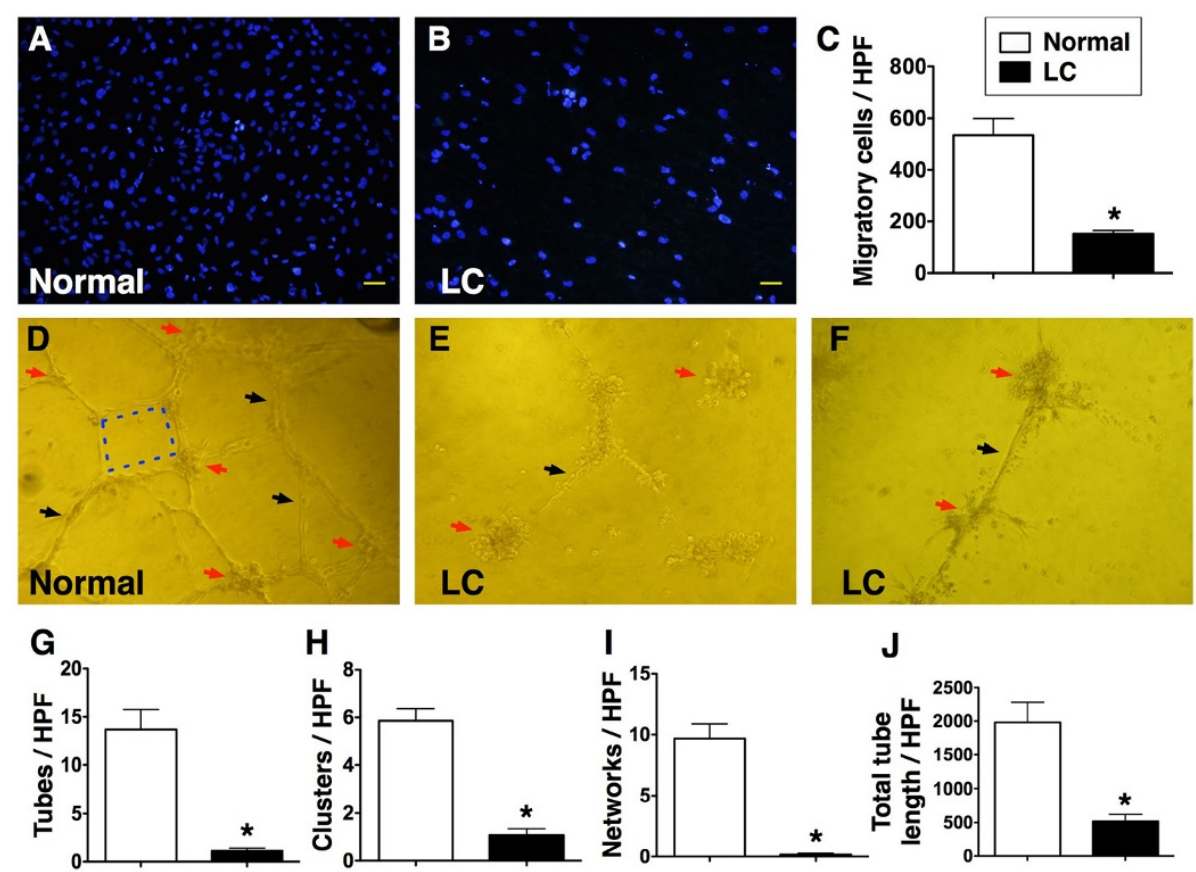

Figure $\mathbf{2}$ In vitro function assessment of endothelial progenitor cells with transwell and matrigel tube formation assay. A to C) The transwell migratory activity of endothelial progenitor cells (EPCs) (100 x) was notably reduced (C) in liver cirrhosis (LC) group than in healthy control group (* vs. normal control, $p<0.0001)(n=6)$. $\mathbf{D}$ to $\mathbf{F}$ ). Illustration of angiogenesis using passages 2 of culturing cells (all pictures were observed under microscope of $400 \mathrm{x}$ ). The cluster formation (red arrows), tubular formation (black arrows) and network formation (blue dot lines) were notably higher in normal control than in LC patients ( $\mathbf{G}$ to $\mathbf{J})$. ( ${ }^{*}$ vs. normal control, all p values $\left.<0.001\right)(n=6)$ High-power field (HPF).

show that the degree of decompensation in liver function was independently correlated to the reduction in circulating number of EPCs (Table 4). Interestingly, Yu et al.. has recently demonstrated dynamic changes in the levels of circulating EPCs in cirrhotic liver using a rat model of HCC [34]. The results of their experiment revealed a gradual increase in the number of circulating EPCs up to 4 weeks after the onset of liver disease, followed by a gradual drop to less than the baseline level at more advanced stage of LC [34]. Accordingly, our findings corroborate those from $\mathrm{Yu}$ et al.[34]. Of importance was that our findings may, therefore, encourage the use of circulating EPC number as an accessory tool in our clinical practice for evaluating the severity of decompensation in liver function.

Although elevations in the serum levels of soluble angiogenic factors are well-documented in the setting of advanced liver disease as in $\mathrm{LC}$ and $\mathrm{HCC}$, the reason for the reduction in circulating number of EPCs in LC patients compared with that in healthy control subjects remains unclear. The results of the present study provided several possible explanations for the phenomenon. First, since the progression from fibrosis to cirrhosis, the end-point of chronic liver diseases, is characterized by a chronic and persistent inflammatory reaction [35.36], we suggest that such chronic inflammation, in addition to stimulating the local release of angiogenic factors by hepatic stellate cells and sinusoidal endothelial cells $[35,36]$, may at the same time suppress the mobilization of EPCs from bone marrow into the circulation. Our hypothesis is supported by the findings of previous studies $[16,17]$ showing a significant decrease in the circulating number of EPCs in patients with hypertension, hypercholesterolemia, current smoking, and diabetes mellitus that are well-known risk factors for coronary artery disease and indexes of chronic inflammatory disease. Second, substantial reduction in circulating EPCs has also reported in patients with decompensated heart failure [19] that is believe to be attributable to an exhaustion of the sources of EPCs. We propose that decompensated heart failure and decompensated LC are two sides of the same coin. Consistently, $50 \%$ of our patients have been identified to have decompensated LC. This proposed mechanism is supported by the findings from both experimental [34] and clinical observational [19] studies.

Surprisingly, contrary to the findings of the present study, a recent investigation [25] demonstrated a higher circulating level of EPCs (i.e. triple staining of the surface EPC marker: CD34/KDR/AC133) in patients with HCC than in those with LC. Moreover, that study also showed a higher number of circulating EPCs in LC patients than that in normal controls. Although the 
discrepancy in results between ours and those from that study [25] seems paradoxical, there may be three possible explanations. First, the treatment status of the patients was different in the two studies. While the previous report enrolled patients waiting for HCC treatment, the current study recruited patients with HCC after treatment, including percutaneous ethanol injection, radiation therapy, radiofrequency ablation, liver lobectomy, or chemotherapy. Second, the degree of decompensation in liver function and the staging for advanced LC may not be identical between the two studies. Finally, differences in sample size, exclusion criteria, methodology for identification of EPCs (CD34/KDR/ AC133 in the recent study [25] vs. CD31/CD34, KDR/ CD34 and CXCR4/CD34 in the present study) may also contribute to the discrepancy in results between the two studies. Another interesting finding is that, as compared with LC patients without HCC, those with HCC had significantly higher circulating number of $\mathrm{E}_{3}$. Besides, LC patients with HCC also had notably higher plasma level of VEGF compared to that in healthy control subjects. In terms of subgroup analysis, therefore, our results were comparable to those of the recent report [25].

The role of SDF- $1 \alpha$ has been established as the ligand of CXCR4+ cells. It has also been reported to play an essential role in mobilizing EPCs from bone marrow to circulation to reach ischemic tissue/organ for angiogenesis and organ repair/regeneration [15]. According to a recent report from Hong et al. [35], activation of CXCR4 receptor by SDF-1 $\alpha$ is profibrogenic in the liver through activation of hepatic stellate cells. In addition to the elevated number of circulating cells with $E_{3}$ in LC patients with HCC than in LC patients without $\mathrm{HCC}$, another interesting finding in the present study is the substantial increase in plasma level of SDF- $1 \alpha$ in LC patients with HCC compared to that in those LC patients without HCC and healthy subjects. Therefore, the results from this clinical observational investigation further strengthen those from Hong's in vivo and in vitro studies [35].

The most important finding in the current study is that, compared with that in the healthy subjects, the capacity for angiogenesis (i.e. cluster, network and tubular formations) was markedly impaired in LC patients. Besides, the transwell migratory ability of EPCs was significantly reduced in LC patients compared to that of the healthy controls. These novel findings implicate that not only was the number of circulating EPCs notably lower in LC patients, but the function of circulating EPCs was also remarkably impaired in this patient population.

Previous studies by other authors and those from our group have identified an association between enhanced systemic cytokine levels, inflammatory responses, and cellular apoptosis after myocardial infarction [33,36]. One of the principal findings in the present study is that MCA in circulation was substantially elevated in LC patients than that in healthy subjects. Additionally, late MCA was further increased in Child's class B and C patients than those in class $\mathrm{A}$. Taking into consideration the fact that chronic liver disease is a process of persistent inflammation, our findings (i.e. MCA) are believed to be the results of chronic inflammatory process in these patients. In this way, these distinctive findings are consistent with those from previous studies [33,36].

In this study, EV bleeding was significantly and independently correlated to an increased number of circulating EPCs. This finding suggests that EV bleeding with acute blood loss may stimulate generation and release of hematopoietic and non-hematopoiectic stem/progenitor cells from bone marrow into the circulation. On the other hand, increased serum level of AST might implicate more severe inflammation and damage in liver parenchyma that might, in turn, suppress the mobilization of EPCs from bone marrow to circulation.

This study has limitations. First, we have no idea regarding the explanation for the finding of chronic hepatitis B as a significant and independent factor associated with an increased number of circulating EPCs in this study. Similar difficulty in explanation is applicable to the negative association between the presence of hepatitis $C$ and a decreased number of circulating EPCs. Second, despite our interesting findings, the sample size of our series is relatively small. All conclusions based on our results, therefore, are tentative and need further support from well-controlled clinical studies of larger scale.

\section{Conclusion}

The results of our study demonstrated that the level, angiogenic ability, and function of circulating EPCs were significantly reduced in cirrhotic patients compared with those in healthy subjects. By contrast, the plasma levels of SDF- $1 \alpha$ and circulating MCA were substantially enhanced in LC patients. These findings may highlight the fact that circulating level of EPCs is reduced not only in patients with risk factors of coronary artery disease but also in patients with LC, especially in those patients with decompensated LC.

\section{Competing interests}

The authors declare that they have no competing interest.

\section{Acknowledgements}

This study was supported by a program grant from Chang Gung Memorial Hospital, Chang Gung University (grant no. CMRPG 890361).

\section{Author details}

${ }^{1}$ Divisions of General Medicine, Kaohsiung Chang Gung Memorial Hospital and Chang Gung University College of Medicine, Kaohsiung, Taiwan. ${ }^{2}$ Basic Science, Nursing Department, Meiho University, Pingtung, Taiwan. ${ }^{3}$ Division of Hepato-gastroenterology, Kaohsiung Chang Gung Memorial Hospital and 
Chang Gung University College of Medicine, Kaohsiung, Taiwan. ${ }^{4}$ Division of Cardiology, Department of Internal Medicine, Kaohsiung Chang Gung Memorial Hospital and Chang Gung University College of Medicine, 123, Ta Pei Road, Niao Sung Hsiang, Kaohsiung Hsien 83301, Taiwan. ${ }^{5}$ Division of Colorectal Surgery, Department of Surgery, Kaohsiung Chang Gung Memorial Hospital and Chang Gung University College of Medicine, Kaohsiung, Taiwan. ${ }^{6}$ Center for Translational Research in Biomedical Sciences, Kaohsiung Chang Gung Memorial Hospital and Chang Gung University College of Medicine, Kaohsiung, Taiwan. 'Department of Emergency Medicine, E-Da Hospital, I-Shou University, Kaohsiung, Taiwan. ${ }^{8}$ Department of Biological Sciences, National Sun Yat-Sen University, Kaohsiung, Taiwan.

\section{Authors' contributions}

All authors have read and approved the final manuscript. L-TC indicates equal contribution in this study compared with the first author. H-WC indicates equal contribution in this study compared with the corresponding author.

Received: 17 January 2012 Accepted: 18 July 2012

Published: 18 July 2012

\section{References}

1. Asahara T, Murohara T, Sullivan A, Silver M, van der Zee R, Li T, Witzenbichler B, Schatteman G, Isner JM: Isolation of putative progenitor endothelial cells for angiogenesis. Science 1997, 275:964-967.

2. Miller-Kasprzak E, Jagodzinski PP: Endothelial progenitor cells as a new agent contributing to vascular repair. Arch Immunol Ther Exp 2007, 55:247-259.

3. Yip HK, Chang LT, Sun CK, Sheu JJ, Chiang CH, Youssef AA, Lee FY, Wu CJ, Fu M: Autologous transplantation of bone marrow-derived endothelial progenitor cells attenuates monocrotaline-induced pulmonary arterial hypertension in rats. Crit Care Med 2008, 36:873-880.

4. Kalka C, Masuda H, Takahashi T, Kalka-Moll WM, Silver M, Kearney M, Li T, Isner JM, Asahara T: Transplantation of ex vivo expanded endothelial progenitor cells for therapeutic neovascularization. Proc Natl Acad Sci USA 2000, 97:3422-3427.

5. Tendera M, Wojakowski W, Ruzyllo W, Chojnowska L, Kepka C, Tracz W, Musialek P, Piwowarska W, Nessler J, Buszman P, et al: Intracoronary infusion of bone marrow-derived selected CD34 + CXCR4+ cells and non-selected mononuclear cells in patients with acute STEMI and reduced left ventricular ejection fraction: results of randomized, multicentre Myocardial Regeneration by Intracoronary Infusion of Selected Population of Stem Cells in Acute Myocardial Infarction (REGENT) Trial. Eur Heart J 2009, 30:1313-1321.

6. Wang S, Cui J, Peng W, Lu M: Intracoronary autologous CD34+ stem cell therapy for intractable angina. Cardiology 2010, 117:140-147.

7. Kocher AA, Schuster MD, Bonaros N, Lietz K, Xiang G, Martens TP, Kurlansky PA, Sondermeijer H, Witkowski P, Boyle A, et al: Myocardial homing and neovascularization by human bone marrow angioblasts is regulated by IL-8/Gro CXC chemokines. J Mol Cell Cardiol 2006, 40:455-464.

8. Huang PH, Chen YH, Wang CH, Chen JS, Tsai HY, Lin FY, Lo WY, Wu TC, Sata M, Chen JW, Lin SJ: Matrix metalloproteinase-9 is essential for ischemia-induced neovascularization by modulating bone marrowderived endothelial progenitor cells. Arterioscler Thromb Vasc Biol 2009, 29:1179-1184

9. Yip HK, Tsai TH, Lin HS, Chen SF, Sun CK, Leu S, Yuen CM, Tan TY, Lan MY, Liou CW, et al: Effect of erythropoietin on level of circulating endothelial progenitor cells and outcome in patients after acute ischemic stroke. Crit Care 2011, 15:R40.

10. Vasa M, Fichtlscherer S, Adler K, Aicher A, Martin H, Zeiher AM, Dimmeler S Increase in circulating endothelial progenitor cells by statin therapy in patients with stable coronary artery disease. Circulation 2001, 103:2885-2890,

11. Wang CH, Verma S, Hsieh IC, Chen YJ, Kuo LT, Yang NI, Wang SY, Wu MY, Hsu CM, Cheng CW, Cherng WJ: Enalapril increases ischemia-induced endothelial progenitor cell mobilization through manipulation of the CD26 system. J Mol Cell Cardiol 2006, 41:34-43.

12. Yeh KH, Sheu J, Lin YC, Sun CK, Chang LT, Kao YH, Yen CH, Shao PL, Tsai TH, Chen YL, et al: Benefit of combined extracorporeal shock wave and bone marrow-derived endothelial progenitor cells in protection against critical limb ischemia in rats. Crit Care Med 2012, 40:169-177.

13. Ergün $\mathrm{S}$, Hohn HP, Kilic N, Singer BB, Tilki D: Endothelial and hematopoietic progenitor cells (EPCs and HPCs): hand in hand fate determining partners for cancer cells. Stem Cell Rev 2008, 4:169-177.

14. Gao D, Nolan DJ, Mellick AS, Bambino K, McDonnell K, Mittal V: Endothelial progenitor cells control the angiogenic switch in mouse lung metastasis. Science 2008, 319:195-198.

15. Imanishi T, Tsujioka H, Akasaka T: Endothelial progenitor cells dysfunction and senescence: contribution to oxidative stress. Curr Cardiol Rev 2008, 4:275-286.

16. Shantsila E, Watson T, Lip GY: Endothelial progenitor cells in cardiovascular disorders. J Am Coll Cardiol 2007, 49:741-752.

17. Hill JM, Zalos G, Halcox JP, Schenke WH, Waclawiw MA, Quyyumi AA, Finkel T: Circulating endothelial progenitor cells, vascular function, and cardiovascular risk. N Engl J Med 2003, 348:593-600.

18. Yip HK, Chang LT, Chang WN, Lu CH, Liou CW, Lan MY, Liu JS, Youssef AA, Chang HW: Level and value of circulating endothelial progenitor cells in patients after acute ischemic stroke. Stroke 2008, 39:69-74.

19. Valgimigli M, Rigolin GM, Fucili A, Porta MD, Soukhomovskaia O, Malagutti P, Bugli AM, Bragotti LZ, Francolini G, Mauro E, et al: CD34+ and endothelial progenitor cells in patients with various degrees of congestive heart failure. Circulation 2004, 110:1209-1212.

20. Makris M, Garson JA, Ring CJ, Tuke PW, Tedder RS, Preston FE: Hepatitis C viral RNA in clotting factor concentrates and the development of hepatitis in recipients. Blood 1993, 81:1898-1902.

21. But DY, Lai CL, Yuen MF: Natural history of hepatitis-related hepatocellular carcinoma. World J Gastroenterol 2008, 14:1652-1656.

22. Mas VR, Maluf DG, Archer KJ, Yanek KC, Fisher RA: Angiogenesis soluble factors as hepatocellular carcinoma noninvasive markers for monitoring hepatitis $C$ virus cirrhotic patients awaiting liver transplantation. Transplantation 2007, 84:1262-1271.

23. Gadelhak NA, Gadelhak SA, El-Morsi DA, Abdelaziz MM, Abbas AT, El-Emshaty HM: Prognostic significance of three hepatitis markers (p53 antibodies, vascular endothelial growth factors and alpha fetoprotein) in patients with hepatocellular carcinoma. Hepatogastroenterology 2009, 56:1417-1424.

24. Aucejo F, Kim R, Zein N, Quintini C, Uso TD, Lopez R, Eghtesad B, Fung J Miller $C$, Yerian $L$ : Vascular endothelial growth factor receptor 2 expression in non-tumorous cirrhotic liver is higher when hepatoma is beyond Milan criteria. Liver Transp/ 2009, 15:169-176.

25. Sieghart W, Fellner S, Reiberger T, Ulbrich G, Ferlitsch A, Wacheck V, Peck-Radosavljevic M: Differential role of circulating endothelial progenitor cells in cirrhotic patients with or without hepatocellular carcinoma. Dig Liver Dis 2009, 41:902-906.

26. Hung CH, Lu SN, Wang JH, Lee CM, Chen TM, Tung HD, Chen CH, Huang WS, Changchien CS: Correlation between ultrasonographic and pathologic diagnoses of hepatitis $B$ and $C$ virus-related cirrhosis. J Gastroenterol 2003, 38:153-157.

27. Baik SK, Jee MG, Jeong PH, Kim JW, Ji SW, Kim HS, Lee DK, Kwon SO, Kim YJ, Park JW, Chang SJ: Relationship of hemodynamic indices and prognosis in patients with liver cirrhosis. Korean J Intern Med 2004, 19:165-170.

28. Wang $\mathrm{JH}$, Changchien $\mathrm{CS}$, Hung $\mathrm{CH}$, Eng $\mathrm{HL}$, Tung WC, Kee KM, Chen $\mathrm{CH}$, Hu TH, Lee CM, Lu SN: FibroScan and ultrasonography in the prediction of hepatic fibrosis in patients with chronic viral hepatitis. $J$ Gastroenterol 2009, 44:439-446.

29. Kim MY, Baik SK, Yea CJ, Lee IY, Kim HJ, Park KW, Kim HK, Suk KT, Kim JW, $\mathrm{Kim} \mathrm{HS}$, et al: Hepatic venous pressure gradient can predict the development of hepatocellular carcinoma and hyponatremia in decompensated alcoholic cirrhosis. Eur J Gastroenterol Hepatol 2009, 21:1241-1246.

30. Pugh RN, Murray-Lyon IM, Dawson JL, Pietroni MC, Williams R: Transection of the oesophagus for bleeding oesophageal varices. Br J Surg 1973, 60:646-649.

31. Chironi G, Walch L, Pernollet MG, Gariepy J, Levenson J, Rendu F, Simon A Decreased number of circulating CD34 + KDR + cells in asymptomatic subjects with preclinical atherosclerosis. Atherosclerosis 2007, 191:115-120.

32. Chang HW, Leu S, Sun CK, Hang CL, Youssef AA, Hsieh YK, Yang CH, Cheng Cl, Chen SM, Chen CJ, et al: Level and value of circulating endothelial progenitor cells in patients with acute myocardial infarction 
undergoing primary coronary angioplasty: in vivo and in vitro studies. Transl Res 2010, 156:251-263.

33. Tsai TH, Lin YC, Sun CK, Chung SY, Chai HT, Yang CH, Chen SM, Hang CL, Chen CJ, Chua S, et al: Prognostic value of circulating dead monocytes in patients with acute st-elevation myocardial infarction undergoing primary percutaneous coronary intervention. Cardiology 2010 117:131-139.

34. Yu DC, Chen J, Sun XT, Zhuang LY, Jiang CP, Ding YT: Mechanism of endothelial progenitor cell recruitment into neo-vessels in adjacent non-tumor tissues in hepatocellular carcinoma. BMC Cancer 2010, 10:435.

35. Hong F, Tuyama A, Lee TF, Loke J, Agarwal R, Cheng X, Garg A, Fiel MI, Schwartz M, Walewski J, et al: Hepatic stellate cells express functional CXCR4: role in stromal cell-derived factor-1alpha- mediated stellate cell activation. Hepatology 2009, 49:2055-2067.

36. Zeller M, Korandji C, Guilland JC, Sicard P, Vergely C, Lorgis L, Beer JC, Duvillard L, Lagrost AC, Moreau D, et al: Impact of asymmetric dimethylarginine on mortality after acute myocardial infarction. Arterioscler Thromb Vasc Biol 2008, 28:954-960.

doi:10.1186/1423-0127-19-66

Cite this article as: Chen et al:: Levels and values of circulating endothelial progenitor cells, soluble angiogenic factors, and mononuclear cell apoptosis in liver cirrhosis patients. Journal of Biomedical Science 2012 19:66.

\section{Submit your next manuscript to BioMed Central and take full advantage of:}

- Convenient online submission

- Thorough peer review

- No space constraints or color figure charges

- Immediate publication on acceptance

- Inclusion in PubMed, CAS, Scopus and Google Scholar

- Research which is freely available for redistribution 